\title{
On Certain Topological Structures of Summable Paranormed Sequence Space Defined in Two - Normed Space
}

\author{
Narayan Prasad Pahari \\ Central Department of Mathematics \\ Tribhuvan University, Kirtipur, Kathmandu, Nepal \\ Email-: nppahari@gmail.com
}

\begin{abstract}
The aim of this paper is to introduce and study a new class $\ell(S, \bar{\xi}, \bar{u})$ of sequences with values in 2- Banach space as a generalization of the familiar space of summable sequences $\ell$. We explore some of the preliminary results that characterize the topological linear structure of the class $\ell(S, \bar{\xi}, \bar{u})$ when topologized it with suitable natural paranorm.
\end{abstract}

Keywords: paranormed space, 2- normed space, sequence space, solid space.

\section{INTRODUCTION}

So far, a bulk number of works have been done on various types of paranormed spaces. The concept of paranorm is closely related to linear metric space and its studies on sequence spaces were initiated by Maddox (1969) and many others.

Before proceeding with the main results,we begin with recalling some of the notations and basic definitions that are used in this paper.

Definition 1: A paranormed space $(S, P)$ is a linear space $S$ with zero element $\theta$ together with a function $P: S \rightarrow$ $\boldsymbol{R}^{+}$(called a paranorm on $S$ ) which satisfies the following axioms:

(i) $P(\theta)=0$;

(ii) $P(s)=P(-s)$, for all $s \in S$;

(iii) $P\left(s_{1}+s_{2}\right) \leq P\left(s_{1}\right)+P\left(s_{2}\right)$, for all $s_{1}, s_{2} \in S$; and

(iv) Scalar multiplication is continuous i.e., if $<\gamma_{n}>$ is a

sequence of scalars with $\gamma_{n} \rightarrow \gamma$ as $n \rightarrow \infty$ and $<s_{n}>$ is a sequence of vectors with $P\left(s_{n}-s\right) \rightarrow 0$ as $n \rightarrow \infty$, then $P\left(\gamma_{n} s_{n}-\gamma s\right) \rightarrow 0$ as $n \rightarrow \infty$. Note that the continuity of scalar multiplication is equivalent to

(i) if $P\left(s_{n}\right) \rightarrow 0$ and $\gamma_{n} \rightarrow \gamma$ as $n \rightarrow \infty$, then $P\left(\gamma_{n} s_{n}\right) \rightarrow 0$ as $n \rightarrow \infty$; and

(ii) if $\gamma_{n} \rightarrow 0$ as $n \rightarrow \infty$ and $s$ be any element in $S$, then $P\left(\gamma_{n} s\right) \rightarrow 0$, see Wilansky (1978).
A paranorm is called total if $P(s)=0 \Rightarrow s=\theta$, see Wilansky (1978).

The studies of paranorms on sequence spaces were initiated by Maddox (1969) and many others. Parasar and Choudhary (1994), Bhardwaj and Bala 2007), Khan (2008), Basariv and Altundag (2009), Tiwari and Srivastava (2010), Pahari $(2011,2013,2013,2014$ \& 2014), and many others further studied various types of paranormed sequence spaces and function spaces.

Definition 2: Let $S$ be a linear space of dimension $>1$ over $\boldsymbol{K}$, the field of real or complex numbers. A 2 - norm on $S$ is a real valued function $\|.,$.$\| on S \times S$ satisfying the following conditions:

(i) $\|s, t\| \geq 0$ and $\|s, t\|=0$ if and only if $s$ and $t$ are linearly dependent;

(ii) $\|s, t\|=\|t, s\|$, for all $s, t \in S$;

(iii) $\|\alpha s, t\|=|\alpha|\|s, t\|$, where $\alpha \in \boldsymbol{K}$ and $s, t \in S$; and

(iv) $\left\|s_{1}+s_{2}, t\right\| \leq\left\|s_{1}, t\right\|+\left\|s_{2}, t\right\|$, for all $s_{1}, s_{2}$ and $t \in S$.

The pair $(S,\|.,\|$.$) is called a 2$-normed space. Thus the notion of 2-normed space is just a two- dimensional analogue of a normed space.

The concept of 2-normed space was initially introduced by S. GÄahler (1963) as an interesting linear generalization of a normed linear space, which was subsequently studied by Iseki (1976), White and Cho (1984), Freese et al. (1992), Freese and Cho (2001) and many others. Recently a lot of 
activities have been started by many researchers to study this concept in different directions, for instances, Gunawan and Mashadi (2001), Açikgöz (2007), Savas (2010), Srivastava and Pahari (2011\& 2013), and others.

Recall that $(S,\|.,\|$.$) is a 2-Banach space if every Cauchy$ sequence $\left\langle s_{n}\right\rangle$ in $S$ is convergent to some $s_{0}$ in $S$. Geometrically, a 2-norm function represents the area of the usual parallelogram spanned by the two associated vectors. As an example, consider $S=\boldsymbol{R}^{2}$, being equipped with $\|\bar{s}, \bar{t}\|=\left|s_{1} t_{2}-s_{2} t_{1}\right|$, where $\bar{s}=\left(s_{1}, s_{2}\right)$ and $\bar{t}=\left(t_{1}\right.$, $\left.t_{2}\right)$. Then $(S,\|.,\|$.$) forms a 2$-normed space and $\|\bar{s}, \bar{t}\|$ represents the area of the parallelogram spanned by the two associated vectors $\bar{s}$ and $\bar{t}$.

Definition 3: Let $(S,\|,\|$,$) be the 2- Normed space$ over the field $\boldsymbol{C}$ of complex numbers and $\bar{\theta}=(\theta, \theta, \theta, \ldots)$ denotes the zero element of $S$. Let $\omega(S)$ denotes the linear space of all sequences $\bar{s}=<s_{k}>$ with $s_{k} \in S, k \geq 1$ with usual coordinate wise operations i.e., for each

$$
\begin{gathered}
\bar{s}=<s_{k}>, \bar{w}=<w_{k}>\in \omega(S) \text { and } \gamma \in \boldsymbol{C}, \\
\bar{s}+\bar{w}=<s_{k}+w_{k}>\text { and } \gamma \bar{s}=<\gamma s_{k}>.
\end{gathered}
$$

We shall denote $\omega(\boldsymbol{C})$ by $\omega$. Any linear subspace of $\omega$ is then called a sequence space.

Further, if $\bar{\gamma}=<\gamma_{k}>\in \omega$ and $\bar{s} \in \omega(S)$ we shall write $\bar{\gamma} \bar{s}=<\gamma_{k} s_{k}>$.

Definition 4: A sequence space $S$ is said to be solid if $\bar{s}=\left\langle s_{k}\right\rangle \in S$ and $\bar{\gamma}=\left\langle\gamma_{k}\right\rangle$ a sequence of scalars with $\left|\gamma_{k}\right| \leq 1$, for all $k \geq 1$, then $\bar{\gamma} \bar{s}=<\gamma_{k} s_{k}>\in S$.

Definition 5: A sequence $\bar{s}=<s_{n}>$ in a linear 2-normed space $S$ is convergent if there is an $s_{0} \in S$ such that $\lim$ $\left\|s_{n}-s, t\right\|=0$, for each $t \in S$.It is said to be a Cauchy if there are $t$ and $w$ in $S$ such that $t$ and $w$ are linearly independent and $\lim _{m, n \rightarrow \infty}\left\|s_{m}-s_{n}, t\right\|=0$ and $\lim _{m, n \rightarrow \infty} \| s_{m}$ $-s_{n}, w \|=0$.

The notion of convergence was introduced by White and Cho (1984). A linear 2-normed space $(S,\|.,\|$.$) is called 2$ Banach space if every Cauchy sequence $<s_{n}>$ in $S$ is convergent to some $s \in S$.

\section{The Class $\ell(S, \bar{\xi}, \bar{u})$ of 2-Normed Space Valued Sequences}

Let $\bar{u}=\left\langle u_{k}>\right.$ and $\bar{v}=\left\langle v_{k}\right\rangle$ be any sequences of strictly positive real numbers and $\xi=<\xi_{k}>$ and $\bar{\mu}=<\mu_{k}>$ be the sequences of non zero complex numbers.

We now introduce the following classes of 2-normed space $S$-valued vector sequences

$\ell(S, \quad, \bar{u})=\left\{\bar{s}=<s_{k}>\in \omega(S)\right.$ satisfying $\sum_{k=1}^{\infty}\left\|\xi_{k} s_{k}, t\right\|$

${ }^{u_{k}}<\infty$, for each $\left.t \in S\right\}$.

In fact, this class is a generalization of the familiar sequence spaces, studied in Pahari (2011, 2013 \& 2014), Srivastava and Pahari (2011, 2011 \& 2013), using 2-norm

\section{RESULTS}

In this section, we shall investigate some results that characterize the linear topological structure of the class $\ell$

$(S, \bar{\xi}, \bar{u})$ of 2 -normed space $S$ - valued sequences by endowing it with suitable natural paranorm. Throughout the work, we denote $z_{k}=\left|\xi_{k} \mu_{k}^{-1}\right|^{u_{k}}$, sup $u_{k}=M$ and for scalar $\alpha, A[\alpha]=\max (1,|\alpha|)$. But when the sequences $<u_{k}$ $>$ and $\left\langle v_{k}\right\rangle$ occur, then to distinguish $M$ we use the notations $M(u)$ and $M(v)$ respectively.

Theorem 1: The space $\ell(S, \bar{\xi}, \bar{u})$ forms a solid.

Proof.

Let $\bar{s}=<s_{k}>\in \ell(S, \bar{\xi}, \bar{u})$. So that for each $t \in S$,

$$
\sum_{k=1}^{\infty}\left\|\xi_{k} s_{k}, t\right\|^{u_{k}}<\infty
$$

Let $\left\langle\gamma_{k}>\right.$ be a sequence of scalars satisfying $\left|\gamma_{k}\right| \leq 1$ for all $k \geq 1$.Then we have

$$
\begin{gathered}
\sum_{k=1}^{\infty}\left\|\xi_{k} \gamma_{k} s_{k}, t\right\|^{u_{k}}=\sum_{k=1}^{\infty}\left|\gamma_{k}\right|^{u_{k}}\left\|\xi_{k} s_{k}, t\right\|^{u_{k}} \\
\leq \sum_{k=1}^{\infty}\left\|\xi_{k} s_{k}, t\right\|^{u_{k}}<\infty,
\end{gathered}
$$

for each $t \in S$. This shows that $\left\langle\gamma_{k} s_{k}>\in \ell(S, \bar{\xi}, \bar{u})\right.$ and hence $\ell(S, \bar{\xi}, \bar{u})$ is normal. 
Theorem 2: For any $\bar{u}=<u_{k}>, \ell(S, \bar{\xi}, \bar{u}) \subset \ell(S, \bar{\mu}, \bar{u})$ if $\lim _{\inf } z_{k}>0$.

Proof.

Assume that $\lim \inf _{k} z_{k}>0$ and $\bar{s}=\left\langle s_{k}>\in \ell(S, \bar{\xi}, \bar{u})\right.$. Then there exist $m>0$ and a positive integer $K$ such that $m\left|\mu_{k}\right|^{u_{k}}<\left|\xi_{k}\right|^{u_{k}}$ for all $k \geq K$ and for each $t \in S$,

$\sum_{k=1}^{\mathrm{K}}\left\|\xi_{k} s_{k}, t\right\|^{u_{k}}<\infty$. Thus for each $t \in S$, we have

$$
\begin{aligned}
\sum_{k=1}^{\mathrm{K}}\left\|\mu_{k} s_{k}, t\right\|^{u_{k}} & \leq \sum_{k=1}^{\mathrm{K}} \frac{\left|\xi_{k}\right|^{u_{k}}}{m}\left\|s_{k}, t\right\|^{u_{k}} \\
& =\frac{1}{m} \sum_{k=1}^{\mathrm{K}}\left\|\xi_{k} s_{k}, t\right\|^{u_{k}}<\infty .
\end{aligned}
$$

This clearly implies that $\bar{s} \in \ell(S, \bar{\mu}, \bar{u})$ and hence $\ell(S, \bar{\xi}, \bar{u}) \subset \ell(S, \bar{\mu}, \bar{u})$. This completes the proof.

Theorem 3: For any $\bar{\xi}=\left\langle\xi_{k}>\right.$, if $u_{k} \leq v_{k}$ for all but finitely many values of $k$, then $\ell(S, \bar{\xi}, \bar{u}) \subset \ell(S, \bar{\xi}, \bar{v})$.

Proof.

Suppose $0<u_{k} \leq v_{k}<\infty$ for all but finitely many values of $k$. Let $\bar{s}=<s_{k}>\in \ell(S, \bar{\xi}, \bar{u})$. Then we have $\sum_{k=1}^{\infty} \| \xi_{k}$ $s_{k}, t \|^{u_{k}}<\infty$, for each $t \in S$.

This shows that there exists $K \geq 1$ such that $\left\|\xi_{k} s_{k}, t\right\|<1$ for all $k \geq K$ and for each $t \in S$.Thus $\left\|\xi_{k} s_{k}, t\right\|^{v_{k}} \leq\left\|\xi_{k} s_{k}, t\right\|$ ${ }^{u_{k}}$ for all $k \geq K$ and for each $t \in S$ and consequently

$$
\sum_{k=1}^{\mathrm{K}}\left\|\xi_{k} s_{k}, t\right\|^{v_{k}} \leq \sum_{k=1}^{\mathrm{K}}\left\|\xi_{k} s_{k}, t\right\|^{u_{k}}<\infty \text {, for each } t \in S
$$

and hence $\bar{s} \in \ell(S, \bar{\xi}, \bar{v})$.This completes the proof of the theorem. The following result is an immediate consequence of Theorems 2 and 3.

Theorem 4: If (i) $\lim \inf _{k} z_{k}>0$; and

$$
\text { (ii) } \quad u_{k} \leq v_{k} \text {,for all but finitely many }
$$

values of $k$, then $\ell(S, \bar{\xi}, \bar{u}) \subset \ell(S, \bar{\mu}, \bar{v})$.
In the following example, we conclude that $\ell(S, \bar{\xi}, \bar{u})$ may strictly be contained in $\ell(S, \bar{\mu}, \bar{v})$ inspite of the satisfaction of both conditions of Theorem 4 .

Example 5: Let $(S, \|$, . $\|)$ be a 2-normed space and consider a sequence $\bar{s}=\left\langle s_{k}>\right.$ defined by $s_{k}=\frac{1}{k^{2 k} s}$, if $k=1,2,3, \ldots$, where $s \in S$ and $s \neq \theta$.

Further, let $u_{k}=\frac{1}{k}$, if $k$ is odd integer, $u_{k}=\frac{1}{k^{2}}$, if $k$ is even integer, $v_{k}=\frac{1}{k} \quad$ for all values of $k, \xi_{k}=3^{k}, \mu_{k}=2^{k}$ for all values of $k$.

Then, $z_{k}=\left|\frac{\xi_{k}}{\mu_{k}}\right|^{u_{k}}=\frac{3}{2}$ or $\left(\frac{3}{2}\right)^{1 / k}$ according as $k$ is odd or even integers and hence $\lim \inf _{k} z_{k}>0$.

Further, $\frac{v_{k}}{u_{k}}=1$, if $k$ is odd integers, $\frac{v_{k}}{u_{k}}=k$, if $k$ is even integers. Therefore $0<u_{k} \leq v_{k}<\infty$ for all $k$.

Hence both the conditions of Theorem 4 are satisfied.

Now for each $t \in S$, we have

$$
\begin{gathered}
\sum_{k=1}^{\infty}\left\|\mu_{k} s_{k}, t\right\|^{v_{k}}=\sum_{k=1}^{\infty}\left\|2^{k} \frac{1}{k^{2 k}} s, t\right\|^{1 / k} \\
\quad=\sum_{k=1}^{\infty} \frac{2}{k^{2}}\|s, t\|^{1 / k} \\
\leq 2 A[\|s, t\|] \sum_{k=1}^{\infty} \frac{1}{k^{2}}<\infty .
\end{gathered}
$$

This shows that $\bar{s} \in \ell(S, \bar{\mu}, \bar{v})$. But on the other hand, let us choose $t \in S$ such that $\|s, t\|=1$. Then for each even integer $k$, we have

$$
\begin{aligned}
\left\|\xi_{k} s_{k}, t\right\|^{u_{k}} & =\left\|3^{k} \frac{1}{k^{2 k}} y, t\right\|^{1 / k^{2}} \\
& =\left(\frac{3}{k^{2}}\right)^{1 / k}\|s, t\|^{1 / k^{2}}>\frac{1}{2} .
\end{aligned}
$$

This implies that $\bar{s} \notin \ell(S, \bar{\xi}, \bar{u})$ and hence the containment of $\ell(S, \bar{\xi}, \bar{u})$ in $\ell(S, \bar{\mu}, \bar{v})$ is strict.

Theorem 6: $\ell(S, \bar{\xi}, \bar{u})$ forms a linear space over the field of complex numbers $C$ if $\left\langle u_{k}>\right.$ is bounded above.

Proof. 
On Certain Topological Structures of Summable Paranormed Sequence Space Defined in Two - Normed Space

Assume that $\sup _{k} u_{k}<\infty$ and $\bar{s}=<s_{k}>, \bar{w}=<w_{k}>$

$\in \ell(S, \bar{\xi}, \bar{u})$.So that for each $t \in S$, we have

$$
\sum_{k=1}^{\infty}\left\|\xi_{k} s_{k}, t\right\|^{u_{k}}<\infty \text { and } \sum_{k=1}^{\infty}\left\|\xi_{k} w_{k}, t\right\|^{u_{k}}<\infty .
$$

Let $0<u_{k} \leq \sup _{k} u_{k}=M, T=\max \left(1,2^{M-1}\right)$ and setting $2 T \max \left(1,|\alpha|^{M}\right) \leq 1$ and $2 T \max \left(1,|\beta|^{M}\right) \leq 1$ and using $|a+b|^{u_{k}} \leq T\left\{|a|^{u_{k}}+|b|^{u_{k}}\right\}$ for all $a, b \in \mathbf{C}$.

Then we have

$$
\begin{aligned}
& \sum_{k=1}^{\infty}\left\|\xi_{k}\left(\alpha s_{k}+\beta w_{k}\right), t\right\|^{u_{k}} \\
\leq & \sum_{k=1}^{\infty}\left[T|\alpha|^{u_{k}}\left\|\xi_{k} s_{k}, t\right\|^{u_{k}}+T|\beta|^{u_{k}}\left\|\xi_{k} w_{k}, t\right\|^{u_{k}}\right] \\
\leq & \sum_{k=1}^{\infty}\left[T A\left[|\alpha|^{M}\right]\left\|\xi_{k} s_{k}, t\right\|^{u_{k}}+T A\left[|\beta|^{M}\right]\left\|\xi_{k} w_{k}, t\right\|^{u_{k}}\right] \\
\leq & \frac{1}{2} \sum_{k=1}^{\infty}\left\|\xi_{k} s_{k}, t\right\|^{u_{k}}+\frac{1}{2} \sum_{k=1}^{\infty}\left\|\xi_{k} w_{k}, t\right\|^{u_{k}}<\infty,
\end{aligned}
$$

for each $t \in S$ and therefore $\alpha \bar{s}+\beta \bar{w} \in \ell((S, \bar{\xi}, \bar{u})$.

This implies that $\ell((S, \bar{\xi}, \bar{u})$ forms a linear space over C.

Theorem 7: If $\ell((S, \bar{\xi}, \bar{u})$ forms a linear space over $\boldsymbol{C}$ then $<u_{k}>$ is bounded above.

Proof.

Suppose that $\ell(S, \bar{\xi}, \bar{u})$ forms a linear space over $C$ but $\sup _{k} u_{k}=\infty$. Then there exists a sequence $\langle k(n)>$ of positive integers satisfying $1 \leq k(n)<k(n+1), n \geq 1$

for which

$$
u_{k(n)}>n, \text { for each } n \geq 1
$$

Now, corresponding to $s_{0} \in S$ and $s_{0} \neq \theta$, we define the sequence $\bar{s}=<s_{k}>$ by $s_{k}=\left\{\begin{array}{l}\xi_{k(n)}{ }^{-1} n^{-2 / u_{k(n)}} s_{0}, \text { if } k=k(n), n \geq 1 \text { and } \\ \theta, \text { otherwise. }\end{array}\right.$

Then for $k=k(n), n \geq 1$, we have

$$
\begin{gathered}
\left.\sum_{k=1}^{\infty}\left\|\xi_{k} s_{k}, t\right\|^{u_{k}}=\sum_{n=1}^{\infty}\left\|n^{-2 / u_{k(n)}} s_{0}, t\right\|^{u_{k(n)}}\right) \\
=\sum_{n=1}^{\infty} \frac{\left\|s, s_{0}\right\|^{u_{k(n)}}}{n^{2}}
\end{gathered}
$$$$
\leq A\left[\left\|s_{0}, t\right\|^{M(u)}\right] \sum_{n=1}^{\infty} \frac{1}{n^{2}}<\infty,
$$

and $\left\|\xi_{k} s_{k}, t\right\|^{u_{k}}=0$, for $k \neq k(n), n \geq 1$,

showing that $\bar{s} \in \quad \ell(S, \bar{\xi}, \bar{u})$.

But on the other hand, let us choose $t_{0} \in S$ such that $\| s_{0}, t_{0}$ $\|=1$. Then for such $t_{0}$ and scalar

$\alpha=4$, for $k=k(n), n \geq 1$, in view of (1) and (2), we have

$$
\begin{aligned}
\sum_{k=1}^{\infty}\left\|\alpha \xi_{k} s_{k}, t_{0}\right\|^{u_{k}} & =\sum_{n=1}^{\infty}\left\|\xi_{k(n)} \alpha s_{k(n)}, t_{0}\right\|^{u_{k(n)}} \\
& =\sum_{n=1}^{\infty}\left\|4 n^{-2 / u_{k(n)}} s_{0}, t_{0}\right\|^{u_{k(n)}} \\
& =\sum_{n=1}^{\infty} \frac{4^{u_{k(n)}}}{n^{2}}\left\|s_{0}, t_{0}\right\| \\
& \geq \sum_{n=1}^{\infty} \frac{4^{n}}{n^{2}}>1 .
\end{aligned}
$$

This shows that $\alpha \bar{s} \notin \ell(S, \bar{\xi}, \bar{u})$, a contradiction. This completes the proof.

The following result is an immediate consequence of Theorems 6 and 7.

Theorem 8: $\ell(S, \bar{\xi}, \bar{u})$ is a linear space over $\boldsymbol{C}$ if and only if $\sup _{k} u_{k}<\infty$.

Let $\bar{u}=<u_{k}>\operatorname{such}$ that $\sup _{k} u_{k}<\infty$ and $\bar{s}=<s_{k}>\in$ $\ell(S, \bar{\xi}, \bar{u})$. We define a real valued function

$P_{\xi, u}(\bar{s})=\left\{\left(\sum_{k=1}^{\infty}\left\|\xi_{k} s_{k}, t\right\|^{u_{k}}\right)^{1 / M}\right.$,for each $\left.t \in S\right\}$.

Throughout the work, $P$ will denote $P_{\xi, u}$ and $\bar{u}=<u_{k}>$, $\bar{v}=<v_{k}>$ such that $\sup _{k} u_{k}<\infty$ and $\sup _{k} v_{k}<\infty$. 
We prove below that $\ell(S, \bar{\xi}, \bar{u})$ with respect to $P$ forms a paranormed space.

Theorem 9: $\ell(S, \bar{\xi}, \bar{u})$ forms a total paranormed -space with respect to $P$.

Proof.

Let $\alpha \in C$ and $\bar{s}=<s_{k}>, \bar{w}=<w_{k}>\in \ell(S, \bar{\xi}, \bar{u})$. Then we can easily verify that $P$ satisfy the following properties of paranorm.

(i) $P(\bar{s}) \geq 0$, and $P(\bar{s})=0$ if and only if $\bar{s}=\bar{\theta}$;

(ii) $P(\bar{s}+\bar{w}) \leq P(\bar{s})+P(\bar{w})$;

(iii) $P(\alpha \bar{s}) \leq A(\alpha) P(\bar{s})$;

(iv) Finally for continuity of scalar multiplication, it is sufficient to show that

(a) $P\left(\bar{s}^{(n)}\right) \rightarrow 0$ and $\gamma_{n} \rightarrow \gamma$ imply $P\left(\gamma_{n} \bar{s}^{(n)}\right) \rightarrow 0$; and

(b) $\gamma_{n} \rightarrow 0$ implies $P\left(\gamma_{n} \bar{s}\right) \rightarrow 0$ for each $\bar{s} \in \ell(S, \bar{\xi}, \bar{u})$.

Now to prove (a) suppose $\left|\gamma_{n}\right| \leq L$ for all $n \geq 1$, then in view of (3), we have

$$
\begin{aligned}
& P\left(\gamma_{n} \bar{s}^{(n)}\right)=\left\{\left(\sum_{k=1}^{\infty}\left\|\gamma_{n} \xi_{k} s_{k}, t\right\|^{u_{k}}\right)^{1 / M}, \text { for each } t \in S\right\} \\
& \leq \sup _{k}\left|\gamma_{n}\right|^{u_{k} / M}\left\{\left(\sum_{k=1}^{\infty}\left\|\xi_{k} s_{k}, t\right\|^{u_{k}}\right)^{1 / M}, \text { for each } t \in S\right\} \\
& \leq A(L) P\left(\bar{s}^{(n)}\right), \text { whence (a) follows. }
\end{aligned}
$$

Next if $\bar{s} \in \ell(S, \bar{\xi}, \bar{u})$, then for $\varepsilon>0$ there exists an integer $K$ such that

$$
\sum_{k=1}^{\mathrm{K}}\left\|\xi_{k} s_{k}, t\right\|^{u_{k}}<\left(\frac{\varepsilon}{2}\right)^{M} \text {, for each } t \in S .
$$

Further if $\gamma_{n} \rightarrow 0$, we can find $N$ such that for $n \geq N$, then for each $t \in S$, we have

$$
\sum_{k=1}^{\mathrm{K}-1}\left|\gamma_{n}\right|^{u_{k}}\left\|\xi_{k} s_{k}, t\right\|^{u_{k}}<\left(\frac{\varepsilon}{2}\right)^{M} \text { and }\left|\gamma_{n}\right| \leq 1 \text {. }
$$

Thus for each $t \in S, P\left(\gamma_{n} \bar{s}\right) \leq\left(\sum_{k=1}^{\mathrm{K}-1}\left\|\gamma_{n} \xi_{k} s_{k}, t\right\|^{u_{k}}\right)^{1 / M}$

$$
+\left(\sum_{k=1}^{\mathrm{K}}\left\|\xi_{k} s_{k}, t\right\|^{u_{k}}\right)^{1 / M}<\varepsilon
$$

for all $n \geq N$, and hence (b) follows.

Theorem 10: If $S$ is a Banach space, then $(\ell(S, \bar{\xi}, \bar{u}), P)$ is complete.

Proof.

We prove the completeness of $\ell(S, \bar{\xi}, \bar{u})$ with respect to the metric $d(\bar{s}, \bar{t})=P(\bar{s}-\bar{t})$.

Let $<\bar{s}^{(n)}>$ be a Cauchy sequence in $\ell(S, \bar{\xi}, \bar{u})$. Then for $0<\varepsilon<1$, there exists $N$ such that for all $n, m \geq N$ and for each $t \in S$, we have $P\left(\bar{s}^{(n)}-\bar{s}^{(m)}\right)$

$=\left(\sum_{k=1}^{\infty}\left\|\xi_{k} s_{k}^{(n)}-\xi_{k} s_{k}^{(m)}, t\right\|^{u_{k}}\right)^{1 / M}<\varepsilon$.

and so for all $n, m \geq N$ and $k \geq 1$ and for each $t \in S$, we have

$\left\|s_{k}^{(n)}-s_{k}^{(m)}, t\right\|<\left|\xi_{k}\right|^{-1} \varepsilon^{M / u_{k}}<\left|\xi_{k}\right|^{-1} \varepsilon$.

This shows that for each $k,<s_{k}^{(n)}>$ is a Cauchy sequence in $S$ and because of completeness of $S, s_{k}^{(n)} \rightarrow s_{k} \in S$ (say) for each $k$. Being a Cauchy sequence $\left\langle s_{k}^{(n)}\right\rangle$ is bounded, i.e. $P\left(s_{k}^{(n)}\right) \leq L$ for some $L>0$ and for all $n \geq 1$. Thus for every $n$ and $r,\left(\sum_{k=1}^{r}\left\|\xi_{k} s_{k}^{(n)}-\xi_{k}, t\right\|^{u_{k}}\right)^{1 / M} \leq L$.

First taking $n \rightarrow \infty$ and then $r \rightarrow \infty$, then for each $t \in S$, $\left(\sum_{k=1}^{\infty}\left\|\xi_{k} s_{k}, t\right\|^{u_{k}}\right)^{1 / M} \leq L$ which implies that $\bar{s}=<s_{k}>$ $\in \ell(S, \bar{\xi}, \bar{u})$.

Now for any $r$, by (4) we have

$\left(\sum_{k=1}^{r}\left\|\xi_{k} s_{k}^{(n)}-\xi_{k} s_{k}^{(m)}, t\right\|^{u_{k}}\right)^{1 / M}<\varepsilon$, for $n, m \geq N$, and so letting $m \rightarrow \infty$ first and then $r \rightarrow \infty$, we get $P\left(\bar{s}^{(n)}-\bar{s}\right)$

$=\left(\sum_{k=1}^{\infty}\left\|\xi_{k} s_{k}^{(n)}-\xi_{k} s_{k}, t\right\|^{u_{k}}\right)^{1 / M} \leq \varepsilon$, for all $n \geq N$ and for each $t \in S$ i.e. $\bar{s}^{(n)} \rightarrow \bar{s}$ in $\ell(S, \bar{\xi}, \bar{u})$, as $n \rightarrow \infty$.

This proves the completeness of $\ell(S, \bar{\xi}, \bar{u})$. 


\section{CONCLUSION}

In the present work, we have studied some of the conditions that typify the topological structures and containment relations of 2-normed space valued summable sequences. In fact, this result can be used for further study to explore other properties of the 2normed space valued sequences and functions.

\section{REFERENCES}

Açikgöz, M. 2007. A review on 2- normed structures. Int. Journal of Math. Analysis. 1(4):187 - 191. Basariv, M. and Altundag, S. 2009. On generalized paranormed statistically convergent sequence spaces defined by Orlicz function. Handawi. Pub. Cor., J. of Inequality and Applications. 2009: 1-13.

Bhardwaj,V.N. and Bala, I. 2007. Banach space valued sequence space $\ell_{M}(X, p)$. Int. J. of Pure and Appl.Maths. 41(5): 617-626.

Freese, R.W. and Cho, Y.J. 2001. Geometry of linear 2normed spaces. Nova Science Publishers, Inc. New York.

Freese, R.W., Cho, Y.J. and Kim, S.S. 1992. Strictly 2convex linear 2-normed spaces. J. Korean Math. Soc. 29( 2) : $391-400$.

GÄahler, S. 1963. 2 -metrische RÄaume undihre to pologische struktur. Math. Nachr. 6:115-148.

Gunawan, H. and Mashadi, H. 2001. On finite dimensional 2-normed spaces. Soochow J. Math. 27: 321-329.

Iseki, K. 1976. Mathematics on two normed spaces. Bull. Korean Math. Soc. 13(2).

Khan,V.A. 2008. On a new sequence space defined by Orlicz functions. Common. Fac. Sci. Univ. Ankseries. 57(2): 25-33.

Maddox, I. J. 1969. Some properties of paranormed sequence spaces. London. J. Math. Soc. 2(1): 316-322.

Pahari, N.P. 2011. On Banach space valued sequence space $l_{\infty}(X, M, \bar{\lambda}, \bar{p}, L)$ defined by Orlicz function. Nepal Jour. of Science and Tech. 12 : 252-259.

Pahari , N.P. 2013. On Certain Topological Structures of Paranormed Orlicz Space $(S((X, \| .||), \Phi, \bar{\alpha}, \bar{u})$, $F$ ) of Vector Valued Sequences, International Jour. Of Mathematical Archive. 4(11): 231241.
Pahari, N.P. 2013. On certain topological structures of 2- normed space valued Orlicz space $c_{0}((S$, $\|.,\|.), \quad \Phi, \bar{u})$ of null sequences. Journal of Global Research in Math. Archives. 1(10) : 6369.

Pahari, N.P. 2014.On certain topological structures of normed space valued generalized Orlicz function space. Int. Jour. of Scientific Engineering and Research (IJSER) 2(1) : 61-66.

Pahari , N.P. 2014. On normed space valued total paranormed Orlicz space of null sequences and its topological structures. Int. Journal of Mathematics Trends and Technology. 6 (Feb): 105-112.

Parashar, S. D. and Choudhary, B. 1994. Sequence spaces defined by Orlicz functions. Indian $J$. Pure Appl. Maths. 25(4): 419-428.

Savas, E. 2010. On some new sequence spaces in 2normed spaces using ideal convergence and an Orlicz function. Hindawi Pub. Corp., Journal of Inequality and Application. 2010:101-115.

Srivastava, J.K. and Pahari, N.P. 2011. On Banach space valued sequence space $l_{M}(X, \bar{\lambda}, \bar{p}, L)$ defined by Orlicz function. South East Asian J.Math. \& Math.Sc. 10(1):39 - 49.

Srivastava , J.K. and Pahari, N.P. 2011. On 2- normed space valued sequence space $l_{M}(X,\|.,\|,. \bar{\lambda}, \bar{p})$ defined by Orlicz function. Proc. of Indian Soc. of Math. and Math. Sc. 6: 243-251.

Srivastava, J.K. and Pahari, N.P. 2013. On 2-Banach space valued paranormed sequence space $c_{0}(X$, $M,\|.,\|,. \bar{\lambda}, \bar{p})$ defined by Orlicz function, Jour. of Rajasthan Academy of Physical Sc. 12(3): 319-338.

Tiwari, R. K. and Srivastava, J. K. 2010. On certain Banach space valued function spaces- II. Math. Forum. 22: 1-14.

White, J.A. and Cho, Y.J. 1984. Linear mappings on linear 2-normed spaces. Bull. Korean Math. Soc. 21(1): $1-6$.

Wilansky, A. 1978. Modern methods in topological vector spaces.Mc Graw_Hill Book Co.Inc. New York. 